\title{
Vibration effects in conditioning of metal powders
}

\author{
Anton Mezenin ${ }^{1}$, Sergey Dmitriev ${ }^{2}$, Margarita Cherkasova ${ }^{3}$ \\ REC “Mekhanobr-Tekhnika”, St. Petersburg, Russia \\ ${ }^{1}$ Corresponding author \\ E-mail: ${ }^{1}$ mezenin_ao@npk-mt.spb.ru, ${ }^{2}$ dmitriev_sv@npk-mt.spb.ru, ${ }^{3}$ cherkasova_mv@npk-mt.spb.ru
}

Received 7 May 2019; accepted 15 May 2019

DOI https://doi.org/10.21595/vp.2019.20782

Check for updates

Copyright (C) 2019 Anton Mezenin, et al. This is an open access article distributed under the Creative Commons Attribution License, which permits unrestricted use, distribution, and reproduction in any medium, provided the original work is properly cited.

\begin{abstract}
The research results are presented for the technology to improve the quality of fine powder materials using vibration effects to produce conditioned commercial products meeting the requirements of additive manufacturing and suitable for application in powder metallurgy and mechanical engineering. The effect of vibration fluidization on the separation of fine metal powder with a particle size of less than 40 microns from submicron particles in a non-uniform electric field using a dielectric barrier discharge, which allows to determine the optimal technological parameters of separation, was studied for the first time. The effect of the operating parameters of the ELKOR-1 corona-electrostatic separator on the behavior of fine-dispersed metal powders with a particle size of less than 160 microns in a non-uniform electric field with high intensity, which allows to determine the optimal separation mode with obtaining standard technological parameters, is investigated.
\end{abstract}

Keywords: metal powder, vibration effects, vibrational fluidization, magnetic separation, electrical separation, additive manufacturing.

\section{Introduction}

High purity powder materials are strongly sought-for by virtually the entire range of production industries utilizing them as raw materials, since the quality of the finished product directly depends on the starting component.

The problem in obtaining high-grade raw materials is similarly urgent for the dynamically developing industry of additive manufacturing, where, due to the high sensitivity of prototyping plants to the quality of raw materials and the high cost and complexity of manufacturing final parts, a set of stringent requirements are imposed on metal powders, the most fundamental of which deal with the grain size and shape.

Particular difficulties in improving the grade of fine metal powders are encountered when their size does not exceed $70 \mu \mathrm{m}$. This is primarily due to the forces of adhesive interaction acting between particles [1]. For their elimination, water is widely used as the dispersion medium, intended to disaggregate the fine material in order to achieve higher grades of the final commercial products. However, the use of process water is associated with significant costs for providing recycled water supply and subsequent drying of the finished product.

This article presents the results of experimental studies demonstrating the fundamental possibility of improving the quality of powder materials with the use of intensive vibration effects, which are, in fact, an alternative to process water in contributing to effective separation of conglomerates formed from finely dispersed metal particles.

The studies were focused on finely dispersed metal powders of different composition and fractions obtained from high alloys. In order to achieve the required grades of fine powders, each powder type was studied separately, depending on the tasks formulated and based on the composition of the initial powder and the impurities contained therein.

\section{Description vibratory fluidization effect}

Mekhanobr-Tekhnika develops technologies and equipment based on the combined physical 
effects of vibration, as well as magnetic and electrical effects, which allows drastically improving the resulting powder grade. In particular, improved fluidity is ensured due to more complete removal of especially fine fractions, including at the submicron level; nonmetallic and ferromagnetic inclusions and non-spherical particles are removed; and the powder materials are sorted by size.

The company is currently researching intensive vibration effects on the material processed, enabling complete elimination of the use of process water as the disaggregating medium and switching to dry technologies with the application of the so-called vibrational fluidization effect [2-6]. The latter consists in the following: the behavior of a bed of a granular medium under vibration is quite accurately characterized by the overload parameter $w$ that predetermines the occurrence of the vibrational fluidization effect. When the condition of $0.3<w<1.3$ is met, mechanical interaction between medium particles in certain intervals of the oscillation period decreases and the particles become mutually mobile. As a result, the mixture is compacted, which is noticeable with the overload parameter of $w>0.3$ and most intense when $w \approx 1.3$.

If the parameter exceeds the value of $w>1.3$, the granular medium bed enters the fluidized state, characterized by the mutual movement of particles of the granular medium, emergence of circulation flows, formation of vortices, etc. In this state, the particles are periodically separated both from the vibrating plane and from each other, which increases the bed volume and causes its vibrational loosening and deflocculation of particle clusters. If the overload parameter is further increased to $w=3$ or higher, the bed is transferred into the state of vibrational suspension.

The approximate boundaries between various bed behavior states for granular media under vibration are shown in Fig. 1.

It may be seen that intensive vibrational fluidization is ensured with the overload parameter of $w \approx 3$. With this overload parameter value, it may be expected that the adhesion forces in combination with the corresponding side effects will not significantly affect the process of separation of fine metal powders from various impurities, including the removal of submicron particles and the destruction of their conglomerates.

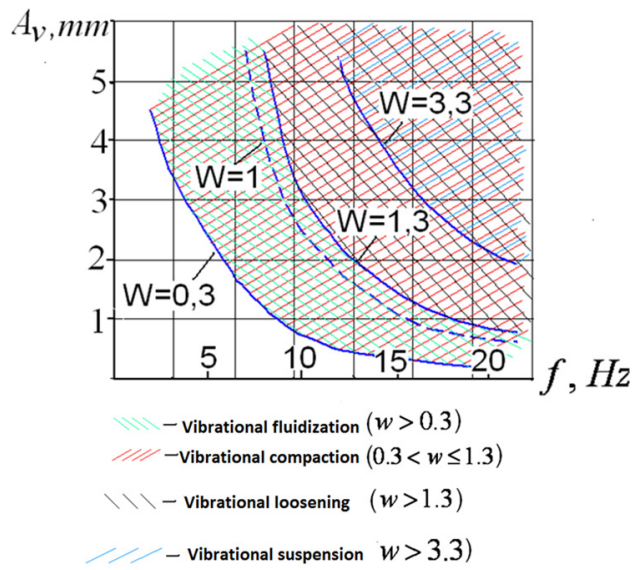

Fig. 1. Approximate boundaries between main bed behavior states for granular media under vibration

\section{Experimental studies on a fine metal powder with a size of 0-40 $\mu \mathrm{m}$}

With the aim of studying the effects of vibrational fluidization on the process indicators of separation, experimental studies were carried out for a fine metal powder with the particle size of 0-40 $\mu \mathrm{m}$, obtained from heat-resistant high-chromium steel. The purpose was to remove submicron particles with the particle size of less than $10 \mu \mathrm{m}$. The studies were carried out using a ZEVS electric drum separator with the dielectric barrier discharge (DBR) [7]. The advantages of DBR in electrical separation are due to the fact that it allows significantly increasing the electric 
field strength and the electrical breakdown voltage and reducing the magnitude of the discharge current. The schematic diagram of the separator is shown in Fig. 2.

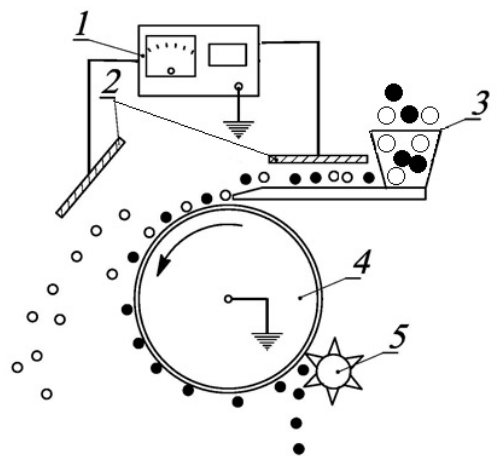

a)

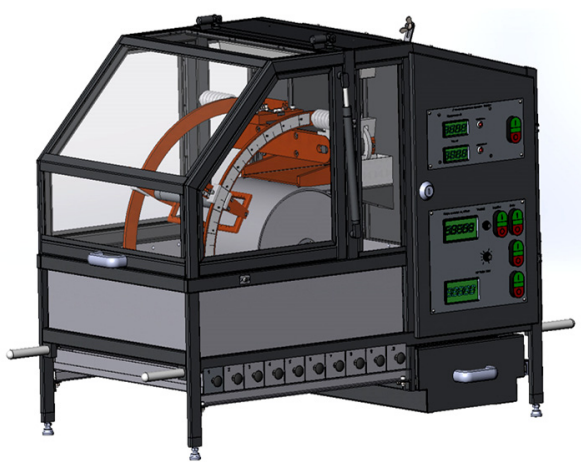

b)

Fig. 2. Schematic diagram of an electric drum separator with vibrational fluidization: 1 - high voltage source, 2 - high voltage electrodes, 3 - vibrating feeder, 4 -drum, 5 - brush

The material to be separated was fed into a vibrating feeder (3) that supplied the feed in the state of vibrational fluidization, which promoted the preliminary separation of conglomerates formed from clustered fine particles, and pre-charged the electrode (2) in the field located above its surface. Particle separation was performed under the action of the field of the second high-voltage electrode (2), located in front of the drum (4). At the same time, the use of a dielectric barrier in the form of a drum coating allowed the particles to maintain the charge obtained on the vibrating feeder and significantly increased the voltage on the high-voltage electrode. Submicron particles, with the particle size of less than $10 \mu \mathrm{m}$, were deposited on the drum and cleaned off with the brush (5), while particles of over $10 \mu \mathrm{m}$ were discharged under the action of the centrifugal force. The results obtained were analyzed using an Analysette 22 NanoTec plus universal laser analyzer, as well as by the yield of the commercial product.

The research results are presented in Fig. 3 and 4, as well as in Table 1.

When analyzing the results obtained, it may be noted that application of the vibrational fluidization effect allows increasing the recovery of particles below $10 \mu \mathrm{m}$ into the corresponding product up to $85.8 \%$ in a single separation procedure. This significantly simplifies cleaning of the middlings, which will ultimately represent the finished product. However, due to the complexity of the separation process, the research in this area is still to be continued.

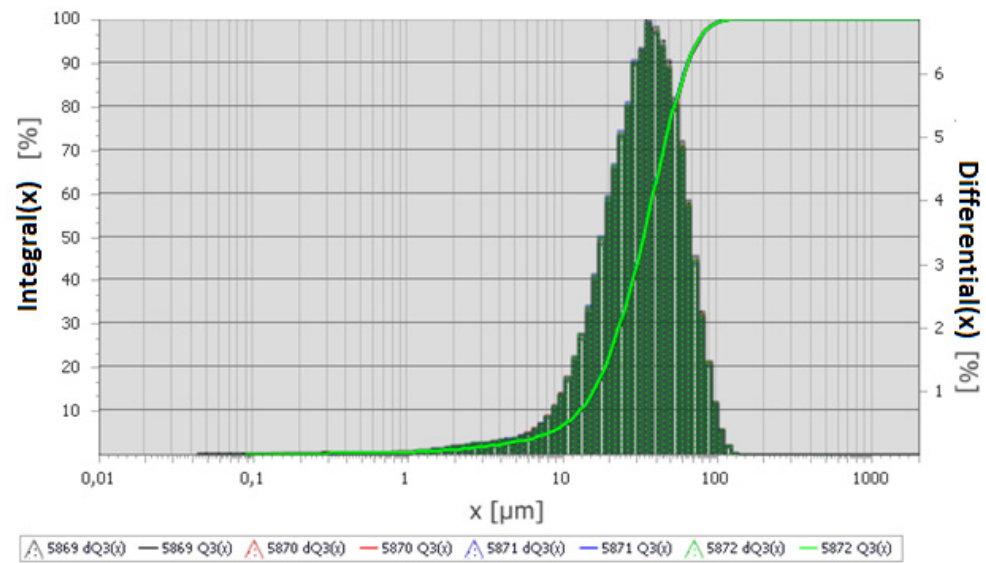

Fig. 3. Metal powder from heat-resistant high-chromium steel before separation 


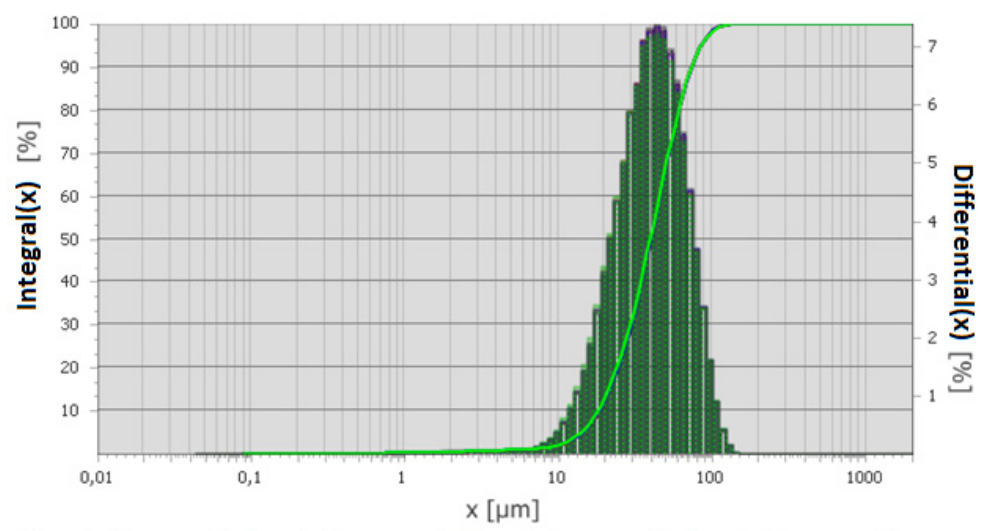

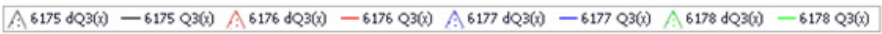

Fig. 4. Metal powder from heat-resistant high-chromium steel after separation

Table 1. Results of metal powder separation using ZEVS electric separator

\begin{tabular}{|c|c|c|c|}
\hline Product & Product yield, $\%$ & Content for class $10 \mu \mathrm{m}, \%$ & Recovery for class $10 \mu \mathrm{m}, \%$ \\
\hline Conductor fraction & 13.4 & 2.8 & 6.7 \\
\hline Middlings & 31.9 & 1.3 & 7.5 \\
\hline Non-conductor fraction & 54.7 & 8.7 & 85.8 \\
\hline Total & 100.0 & 5.6 & 100.0 \\
\hline
\end{tabular}

\section{Experimental studies of fine metal powders with a particle size of 63-160 $\mu \mathrm{m}$ and 64-140 $\mu \mathrm{m}$}

In the study of fine metal powders based on cobalt and nickel, the main task was to remove non-spherical particles (mainly "flat" particles). The particle-size distribution of the cobalt and nickel powders varied between 63-160 $\mu \mathrm{m}$ and 64-140 $\mu \mathrm{m}$, respectively. The cobalt powder, having pronounced ferromagnetic properties, was subjected to separation using a PBSTs-30/5 magnetic drum separator in a non-uniform magnetic field with the magnetic induction of $0.35 \mathrm{~T}$. The feed was supplied into the separation area by a vibrating feeder, where spherical particles of the cobalt powder, being attracted to the drum, were carried over into the magnetic product compartment. Application of the vibrational fluidization effect for the feed material enabled a more uniform distribution of the material upstream of the working area of the separator. Non-spherical particles with less pronounced magnetic properties were discharged by the centrifugal force from the drum into the non-magnetic product compartment. The magnetic product was then subjected to repeated re-cleaning to obtain the commercial product of the required grade. The separation products here and in the subsequent experiments were analyzed visually, using a Bresser Science MTL-201 metallographic microscope, and by the yield of the finished product.

The nickel-based fine powder was subjected to separation using an ELKOR-1 corona-electrostatic separator in a non-uniform electric field with high intensity. The feed material was transported by a vibrating feeder into the material separation zone. Application of the vibrational fluidization effect for the feed supplied by the vibrating feeder, in this case, ensured the most uniform distribution of the material upstream of the working area of the separator and additional tribo-charging of metal particles. Due to the fact that the particles of the material separated differ in shape, they have different electrical properties. When in the effective area of the electric field, spherical particles behave like conductors, while non-spherical particles behave like dielectrics. In order to obtain a positive separation result, an optimal separation regime was selected, including the selection of such parameters as voltage on the high-voltage electrodes, drum rotation frequency, electrode configuration and a set of other parameters to ensure that the feed was supplied in the vibrational fluidization state. The separation results are shown in Fig. 5. 
The studies conducted for the finely dispersed cobalt and nickel powders rendered conditioned commercial products with the commercial yield of $99.2 \%$ and $92.0 \%$, respectively. The visual analysis of the conditioned commercial products obtained during the separation revealed no contaminating impurities.

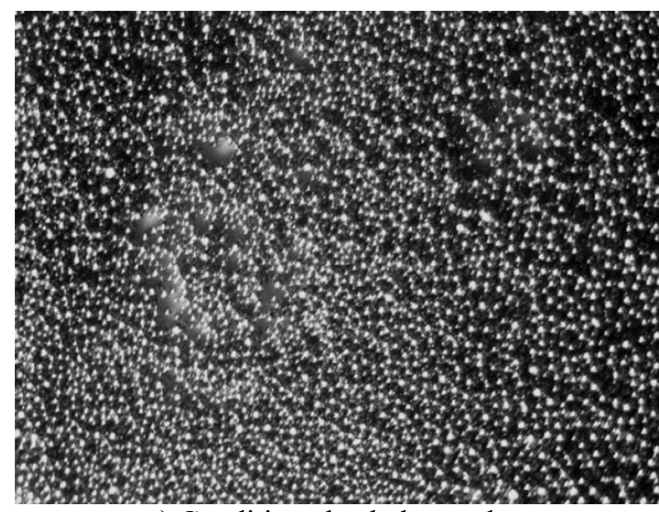

a) Conditioned cobalt powder

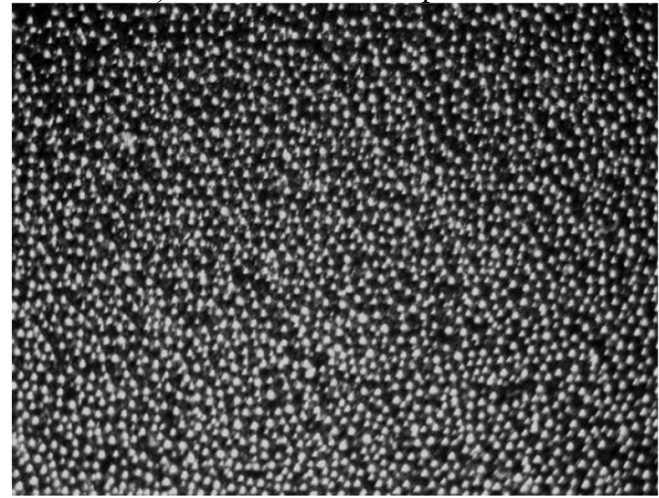

c) Conditioned nickel powder

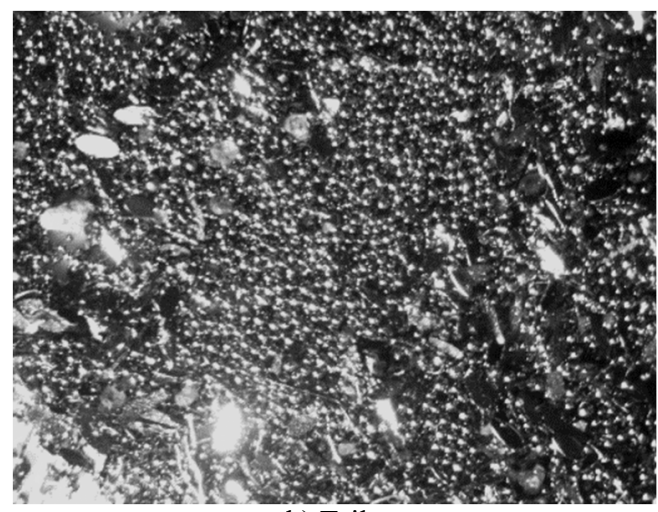

b) Tails

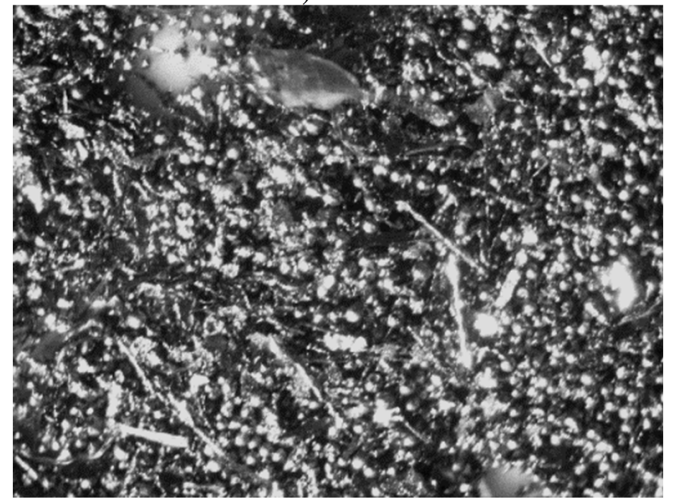

d) Tails

Fig. 5. Results of separation of cobalt and nickel powders by magnetic and electrical separation methods

\section{Conclusions}

The studies have demonstrated the fundamental possibility of obtaining high-purity standard metal powders mainly by the magnetic and electrical methods in combination with vibration effects.

The supply of the feed material in the state of vibrational fluidization contributes to the destruction of conglomerates formed from finely dispersed metal particles and ensures subsequent efficient separation of metal powders with the removal of various types of impurities.

It should be noted that all the equipment used for the research has high performance, low energy consumption and high environmental safety.

\section{Acknowledgements}

The work was carried out jointly with the Peter the Great St. Petersburg Polytechnic University and POLEMA JSC (Tula).

The study was carried out under the grant issued by the Russian Science Foundation (Project No. 17-79-30056). 


\section{References}

[1] Vaisberg L. A., Dmitriev S. V., Mezenin A. O. Manageable magnetic anomalies in mineral processing technology. Gornyi Zhurnal (Mining Journal), Vol. 10, 2017, p. 26-32.

[2] Demidov I. V., Dmitriev S. V., Ivanov K. S., Mezenin A. O. Towards the development of the theory of dry magnetic and electrostatic separation. Obogashchenie Rud (Mineral Processing Journal), Vol. 6, 2018, p. 33-38.

[3] Golovanevskiy V. A., Arsentyev V. A., Blekhman I. I., Vasilkov V. B., Azbel Y. I., Yakimova K. S. Vibration-induced phenomena in bulk granular materials. International Journal of Mineral Processing, Vol. 100, Issues 3-4, 2011, p. 79-85.

[4] Vaisberg L. A., Demidov I. V., Ivanov K. S. Mechanics of granular media under vibration action: the methods of description and mathematical modeling. Obogashchenie Rud (Mineral Processing Journal), Vol. 4, 2015, p. 21-31.

[5] Aranson Igor S., Tsimrimg Lev S. Patterns and collective behavior in granular media: theoretical concepts. Reviews of Modern Physics, Vol. 78, 2006, p. 641.

[6] Jaeger H. M., Nagel S. R., Behringer R. P. Granular solids, liquids, and gases. Reviews of Modern Physics, Vol. 68, 1996, p. 1259.

[7] Dmitriev S. V., Grigoriev I. V. Dielectric barrier discharge application prospects in electrostatic separation. Obogashchenie Rud (Mineral Processing Journal), Vol. 4, 2018, p. 34-38. 